\title{
Cuidadores Familiares de Idosos com a Doença de Alzheimer
}

\author{
Family Caregivers of Seniors with Alzheimer's Disease \\ Cuidadores Familiares de Personas Mayores con \\ Enfermedad de Alzheimer
}

Camila Rodrigues Garcia

Gabriela Cabett Cipolli

Jéssica Pucci dos Santos

Luca Pasquali Freitas

Mayara Cartoni Braz

Deusivania Vieira da Silva Falcão

RESUMO: Este estudo teve como objetivo investigar na perspectiva de cuidadores familiares de idosos com provável/possível diagnóstico da doença de Alzheimer: a reação inicial da família diante do provável/possível diagnóstico da doença de Alzheimer; as principais atividades realizadas com estes idosos; as fontes de auxílio no cuidado e o grau de satisfação em relação a esse auxílio; os sentimentos vivenciados diante da tarefa de cuidar e a dinâmica familiar após a enfermidade. A amostra foi composta por 17 cuidadores familiares que contaram com o auxílio da Associação de Familiares e Amigos dos Idosos (AFAI). Os dados foram coletados por meio de um questionário com perguntas abertas e fechadas. Os resultados demonstraram que a reação inicial dos familiares foi desfavorável diante do provável/possível diagnóstico da DA e com a progressão da doença, os cuidadores vivenciaram sentimentos desfavoráveis diante da tarefa de cuidar, desencadeando mudanças na dinâmica familiar.

Palavras-chave: Doença de Alzheimer; Cuidador; Família. 
ABSTRACT: This study aimed to investigate the perspective of family caregivers of older adults with probable / possible diagnosis of Alzheimer's disease: the initial reaction of the family before the probable / possible diagnosis of Alzheimer's disease; the main activities carried out with these elderly; sources of help in the care and the degree of satisfaction in relation thereto; the feelings experienced on the care task and the family dynamics after illness. The sample consisted of 17 family caregivers who relied on the help of the Association of Relatives and Friends of the Elderly (AFAI). Data were collected through a questionnaire with open and closed questions. The results showed that the initial reaction of the family was unfavorable against the probable / possible diagnosis of AD and with the progression of the disease, caregivers experienced unfavorable feelings about the care task and changes in family dynamics.

Keywords: Alzheimer's disease; Caregiver; Family.

RESUMEN: El objetivo de este estudio fue investigar la perspectiva de los cuidadores familiares de adultos mayores con diagnóstico probable / posible de la enfermedad de Alzheimer: la reacción inicial de la familia antes del probable / posible diagnóstico de la enfermedad de Alzheimer; Las principales actividades realizadas con estos ancianos; Las fuentes de ayuda en el cuidado y el grado de satisfacción en relación con el mismo; Los sentimientos experimentados en la tarea de cuidado y la dinámica familiar después de la enfermedad. La muestra consistió en 17 cuidadores familiares que confiaron en la ayuda de la Asociación de Familiares y Amigos de Ancianos (AFAI). Los datos fueron recogidos a través de un cuestionario con preguntas abiertas y cerradas. Los resultados mostraron que la reacción inicial de la familia fue desfavorable frente al diagnóstico probable / posible de la EA y con la progresión de la enfermedad, los cuidadores experimentaron sentimientos desfavorables sobre la tarea de cuidado y cambios en la dinámica familiar.

Palabras clave: Enfermedad de Alzheimer; Cuidador; Familia.

\section{Introdução}

O envelhecimento populacional pode ser considerado um fenômeno mundial, que traz possibilidades e desafios a serem enfrentados. Nesse cenário, prevê-se o aumento da prevalência de demências, sendo a mais frequente delas a doença de Alzheimer (DA). 
Uma estimativa realizada nos Estados Unidos sinalizou que, em 2050, os casos de DA duplicarão, chegando a uma quantidade aproximada de 13,8 milhões de idosos com a demência (Moon, \& Dilworth-Anderson, 2014).

No Brasil, pela inexistência de pesquisa nacional específica, não dispomos de dados oficiais a respeito do número de pessoas com doença de Alzheimer e, consequentemente, do custo que essa doença representa tanto para a sociedade quanto para as famílias (Machado, 2012).

A DA é uma doença progressiva e degenerativa do cérebro que acomete inúmeras áreas cognitivas resultando em um declínio da capacidade funcional e alterações comportamentais (Kang, et al., 2014). A partir do momento em que o diagnóstico da DA é estabelecido, os familiares ficam tomados de sentimentos variados, tais como: a esperança da cura; a escolha do cuidador; a questão financeira (considerando-se o alto custo do tratamento); e a busca por ajuda (Vilela, \& Caramelli, 2006; Falcão, 2006). Nesse contexto, os idosos demenciados necessitam receber cuidados, os quais são realizados quase sempre no próprio domicílio ou na residência de parentes (Neuman, Dias, \& Falcão, 2016).

A "eleição" do cuidador é amiúde uma das principais escolhas da família, sendo baseada nas necessidades de cuidado e com referencial de gênero - já que na maioria dos casos é a mulher (cônjuge, filha ou nora) que assume este papel - obrigando que ocorram rearranjos familiares. Assim, é comum ocorrerem conflitos nas relações intrafamiliares, que podem ser explicadas pelas condições que o cuidador familiar assumiu ao estabelecer tal função. Nesse cenário, poucas são as famílias que conseguem dividir o trabalho entre seus componentes de uma maneira igualitária, favorecendo a sobrecarga de papéis na pessoa que cuida (Falcão, \& Bucher-Maluschke, 2008, 2009; Freitas, Paula, Soares, \& Parente, 2008; Almeida, 2005).

O termo sobrecarga é utilizado na literatura gerontológica nacional para descrever os efeitos negativos da tarefa de cuidar no cuidador (Figueiredo, \& Sousa, 2008), e este pode ser definido como um estressor, caracterizado pelas situações em que as famílias são forçadas a ajustar ou desenvolver novas estratégias de lidar com a pessoa doente e seus sintomas (Gratão, 2006; 2010). Essa sobrecarga relacionada com a tarefa de cuidar geralmente deve-se à qualidade ou quantidade de demanda que ultrapassam a capacidade de desempenho, por insuficiência técnica ou de tempo, e que a pressão e a responsabilidade no trabalho propiciam, principalmente, o aparecimento da exaustão emocional (Maslach, Schaufeli, \& Leiter, 2001). 
Schulz e Beach (1999) relataram que, além das questões físicas, familiares e psicológicas, tais como estresse, apatia, e tristeza crônica, os cuidadores familiares tendem a enfrentar diversas dificuldades financeiras e sociais durante o processo demencial. Distúrbios de comportamento são um dos problemas que podem emergir durante a evolução da doença. Contudo, o ato de cuidar, também, pode gerar benefícios para quem o executa, tais como a oportunidade de reflexão, autoavaliação, crescimento (Falcão, \& BucherMaluschke, 2009), aprendizado, consciência e perdão (Oliveira, Ferreira, Fonseca, \& Paes, 2016). Conforme Neri e Sommerhalder (2002), a despeito da sobrecarga de papéis, muitos cuidadores se sentem gratificados com os aspectos que envolvem a atividade de cuidar. Nesse cenário, faz-se mister desenvolver pesquisas na área com o intuito de favorecer intervenções clínicas que promovam a coesão familiar e o bem-estar dos idosos e seus cuidadores.

A avaliação da qualidade do cuidado, os modelos educativos para cuidados em saúde e a qualidade de vida dos cuidadores e idosos com Alzheimer são inquietações da comunidade científica mundialmente (Resende, \& Dias, 2008; Leite, Menezes, Lyra, \& Araújo, 2014). Partindo-se dessas afirmações, este estudo teve como objetivo investigar na perspectiva de cuidadores familiares de idosos com provável/possível diagnóstico da doença de Alzheimer: a reação inicial da família diante do provável/possível diagnóstico da doença de Alzheimer; as principais atividades realizadas com estes idosos; as fontes de auxílio no cuidado e o grau de satisfação em relação a esse auxílio; os sentimentos vivenciados diante da tarefa de cuidar; e a dinâmica familiar após a enfermidade.

\section{Método}

Trata-se de uma pesquisa descritiva e exploratória. Participaram 17 cuidadores familiares de pessoas idosas e 17 idosos com DA que frequentavam a Associação de Familiares e Amigos dos Idosos (AFAI), localizada na cidade de São Paulo, SP. Os critérios de inclusão dos sujeitos para pesquisa foram os seguintes: a) ser familiar cuidador de idosos com provável/possível diagnóstico de Alzheimer; b) participar das atividades desenvolvidas pela AFAI.

O instrumento utilizado foi um questionário composto por questões abertas e fechadas, elaborado a partir de uma revisão bibliográfica. Inicialmente, houve um contato com a diretoria da AFAI, solicitando-se permissão para a aplicação da pesquisa. 
Após autorização, foi feito um contato com os familiares para informar sobre o estudo e convidar para participarem deste. Após a anuência dos sujeitos selecionados, apresentou-se o Termo de Consentimento Livre e Esclarecido, respeitando-se as questões éticas. O questionário foi entregue aos cuidadores por meio de um envelope lacrado. Cada cuidador respondia com o próprio punho e individualmente o instrumento. Em seguida, devolvia-o aos pesquisadores.

Quanto à análise dos dados, no caso das questões qualitativas, estas foram analisadas por meio da técnica de Bardin (1977/2000), e se constituiu em três períodos: a pré-análise, a exploração do material e o tratamento dos resultados, obedecendo às regras de: (a) exaustividade - esgotando-se a totalidade da comunicação, não omitindo nenhuma informação; (b) representatividade - representou-se o universo da amostra investigada; (c) homogeneidade - os dados referiram-se ao mesmo tema, foram obtidos por técnicas iguais e aplicadas por indivíduos semelhantes; (d) pertinência - as entrevistas foram adaptadas aos objetivos da pesquisa; e (e) exclusividade - um elemento não foi classificado em mais de uma categoria.

A análise da informação codificada favoreceu a verificação das ocorrências de cada categoria, a identificação de subcategorias, e a extração dos trechos das entrevistas correspondentes a elas. $\mathrm{O}$ tratamento dos resultados obtidos $\mathrm{e}$ as interpretações compreenderam a frequência simples das unidades de análise. A ponderação da frequência de cada unidade traduziu um caráter quantitativo (dimensão) e qualitativo (direção). A direção dos conteúdos analisados foi favorável (aspectos positivos das afirmações), desfavorável (aspectos negativos das informações) ou neutra (conteúdo indefinido, vago, indeterminado, indiferente ou imparcial, que não expressava partido nem a favor nem contra). Nesse sentido, foi realizada uma análise qualitativa e quantitativa das entrevistas. Visando-se a uma melhor compreensão, apresenta-se o quadro 1 dos resultados. Para os dados quantitativos, utilizou-se a estatística descritiva (frequência e média) para realizar a análise.

\section{Resultados}

A idade dos participantes variou entre 34-78 anos, sendo que 5 (29\%) eram do sexo masculino; e $12(71 \%)$ do sexo feminino. 
A média de idade foi de 61,41 anos. O sexo masculino apresentou uma média de idade de aproximadamente 64 anos; e o sexo feminino de aproximadamente 60 anos.

Em relação ao estado civil, 59\% eram casados; $17 \%$ solteiros; $12 \%$ união estável; $6 \%$ viúvos; e $6 \%$ não responderam. O nível de escolaridade da amostra total foi de $47 \%$ com nível superior completo; $35 \%$ com médio completo; $12 \%$ com superior incompleto; e $6 \%$ com pósgraduação. A renda máxima observada foi de 11 salários mínimos com 12\% do total; enquanto o mínimo foi de 2-5 salários mínimos com 17\% da amostra. Dentre os cuidadores 6 (35\%) estava aposentada; 5 (29\%) era dona de casa; e os demais exerciam profissão fora do lar em tempo parcial.

Já os idosos com DA tinham a idade entre 70-91 anos, sendo que 4 (23\%) eram do sexo masculino; e $13(77 \%)$ eram do sexo feminino. A média de idades entre eles era de 82 anos. Em relação ao estado civil, 59\% eram viúvos; $23 \%$ casados; $12 \%$ em união estável; $6 \%$ não responderam. Quanto ao nível de escolaridade, $47 \%$ concluiu o primário; $17 \%$ o ensino médio completo; $12 \%$ o nível superior incompleto; $6 \%$ o primário incompleto. Além disso, $47 \%$ dos idosos moravam com uma pessoa; $30 \%$ com três pessoas; $17 \%$ duas pessoas; e $6 \%$ com quatro pessoas.

O tempo de provável/possível diagnóstico da doença foi classificado em quatro categorias: de 0 a 3 anos; de 4 a 6 anos; de 7 a 9 anos; e de 10 anos ou mais. Detectou-se que 36\% (6) dos idosos possuíam o provável/possível diagnóstico há 10 anos ou mais; $23 \%$ (4) há 0 a 3 anos; 23\% (4) há 4 a 6 anos; $12 \%$ (2) entre sete a nove anos; e 6\% (1) não respondeu.

Quanto à reação inicial da família diante do provável/possível diagnóstico da doença de Alzheimer no idoso, constatou-se, no geral, que a reação inicial dos familiares foi desfavorável diante do provável/possível diagnóstico da DA, conforme a tabela 1.

Tabela 1: Reação inicial da família diante do diagnóstico/ suspeita da doença de Alzheimer no idoso

\begin{tabular}{lcc}
\hline \begin{tabular}{c} 
Reação inicial da família diante do \\
provável/possível diagnóstico da \\
\multicolumn{1}{c}{ doença de Alzheimer }
\end{tabular} & \multicolumn{2}{c}{ Frequência das unidades de análise } \\
\cline { 2 - 3 } & Absoluta & Percentual \\
\hline Reação inicial desfavorável & 24 & 70 \\
Reação inicial favorável & 5 & 15 \\
Reação inicial neutra & 5 & 15 \\
\hline \multicolumn{1}{c}{ Total } & 34 & 100 \\
\hline
\end{tabular}




\section{As principais atividades realizadas pelos cuidadores direcionadas aos idosos} foram divididas em duas categorias: atividades instrumentais e atividades afetivas. Em geral, as atividades instrumentais eram as mais realizadas pelos familiares. Cuidar da alimentação e da medicação do idoso, assim como levá-lo ao médico, eram as principais atividades instrumentais realizadas pelos familiares. As atividades afetivas, como conversar e ouvir, dar carinho, e dar apoio emocional, também eram realizadas pelos cuidadores. Para maiores informações, ver a Tabela 2.

Tabela 2: Principais atividades realizadas pelos cuidadores familiares

\begin{tabular}{lccccc}
\hline \multicolumn{1}{c}{ Atividades Instrumentais } & \multicolumn{4}{c}{ Atividades Afetivas } \\
\hline \multicolumn{1}{c}{ Atividade } & n & $\%$ & Atividade & n & $\%$ \\
\hline $\begin{array}{l}\text { Cuidar da } \\
\text { alimentação e da } \\
\text { medicação }\end{array}$ & 17 & $100 \%$ & Conversar/Ouvir & 15 & $88 \%$ \\
Levar ao médico & 17 & $100 \%$ & Dar carinho & 15 & $88 \%$ \\
$\begin{array}{l}\text { Controlar/Orientar } \\
\text { Cuidar da higiene }\end{array}$ & 16 & $94 \%$ & Dar apoio emocional & 15 & $88 \%$ \\
pessoal & 14 & $82 \%$ & & & \\
$\begin{array}{l}\text { Passear/Realizar } \\
\text { caminhadas }\end{array}$ & 12 & $71 \%$ & & & \\
$\begin{array}{l}\text { Ajudar nas tarefas } \\
\text { domésticas }\end{array}$ & 9 & $53 \%$ & & & \\
$\begin{array}{l}\text { Ajudar } \\
\text { financeiramente }\end{array}$ & 7 & $41 \%$ & & & \\
$\begin{array}{l}\text { Levar e buscar o } \\
\text { idoso na AFAI e em } \\
\text { outros lugares }\end{array}$ & 3 & $18 \%$ & & & \\
$\begin{array}{l}\text { Acompanhar nas } \\
\text { atividades do } \\
\text { Centro-dia (AFAI) }\end{array}$ & 1 & $6 \%$ & & & \\
$\begin{array}{l}\text { Visitar outros } \\
\text { familiares }\end{array}$ & 1 & $6 \%$ & & & \\
\hline
\end{tabular}


As principais fontes de auxílio no cuidado relatadas pelos familiares foram: as instituições, tais como a AFAI, correspondendo ao auxílio para 15 familiares (88\%); outros familiares, correspondendo a um auxílio para 14 famílias (82\%); empregada doméstica e cuidadora formal, correspondendo, cada uma, a um auxílio para 1 familiar (6\%). No entanto para 1 familiar, ninguém o auxilia no cuidado.

Quanto ao grau de satisfação em relação ao auxílio no cuidado recebido, foi visto que a maioria dos familiares apresentou um grau de satisfação positivo, em relação ao auxílio recebido no cuidado do idoso com Alzheimer. Os resultados apontaram que 35\% (6) dos familiares consideraram esse suporte satisfatório; e 29\% (5) muito satisfatório. Contudo, $24 \%$ (4) dos familiares consideraram o suporte recebido como pouco satisfatório; e $6 \%$ (1) nada satisfatório. Apenas um familiar não se posicionou a respeito dessa questão.

No que se refere à gestão do tempo (há tempo para cuidar de si próprio e do idoso com Alzheimer?) foi visto que a maioria dos familiares $(71 \% ; 12)$, afirmou não ter tempo para cuidar de si mesmo, devido à quantidade de horas despendida no cuidado do idoso com DA. Apenas 5 (29\%) dos cuidadores não considerou que o cuidado para com o idoso interferiu no tempo dedicado para si mesmo.

No que se refere aos sentimentos do cuidador em relação à tarefa de cuidar, notou-se que existem mais sentimentos desfavoráveis do que favoráveis, conforme a tabela 3.

Tabela 3: Sentimentos do cuidador diante do papel de cuidar do idoso com DA

\begin{tabular}{lcc}
\hline $\begin{array}{c}\text { Sentimento do cuidador familiar diante do papel de } \\
\text { cuidar do idoso com Alzheimer }\end{array}$ & \multicolumn{2}{c}{$\begin{array}{c}\text { Frequência das } \\
\text { unidades de análise }\end{array}$} \\
\cline { 2 - 3 } & Absoluta & Percentual \\
Sentimentos desfavoráveis & 34 & $87 \%$ \\
Sentimentos favoráveis & 4 & $13 \%$ \\
\hline Total & 39 & 100 \\
\hline
\end{tabular}


Com relação à dinâmica familiar, detectou-se que, após o provável/possível diagnóstico da doença de Alzheimer, a maioria (16; 94\%) dos familiares relatou ter havido mudanças na dinâmica familiar. Assim sendo, apenas 1 cuidador não relatou alteração na dinâmica da família. Outrossim, 47\% (8) dos familiares afirmaram que o relacionamento com outros membros da família foi algumas vezes atingido desfavoravelmente em decorrência do processo demencial do idoso; e $29 \%$ (5) que essa relação era sempre atingida de uma maneira desfavorável. Apenas $12 \%$ (2) relatou que raramente o relacionamento era afetado devido à doença; e 12\% (2) não percebia mudança no relacionamento familiar após a enfermidade do idoso.

A seguir, apresenta-se um quadro, sintetizando as categorias e subcategorias sinalizadas nas tabelas 2 e 3 , ilustrando-se com exemplos das falas dos sujeitos.

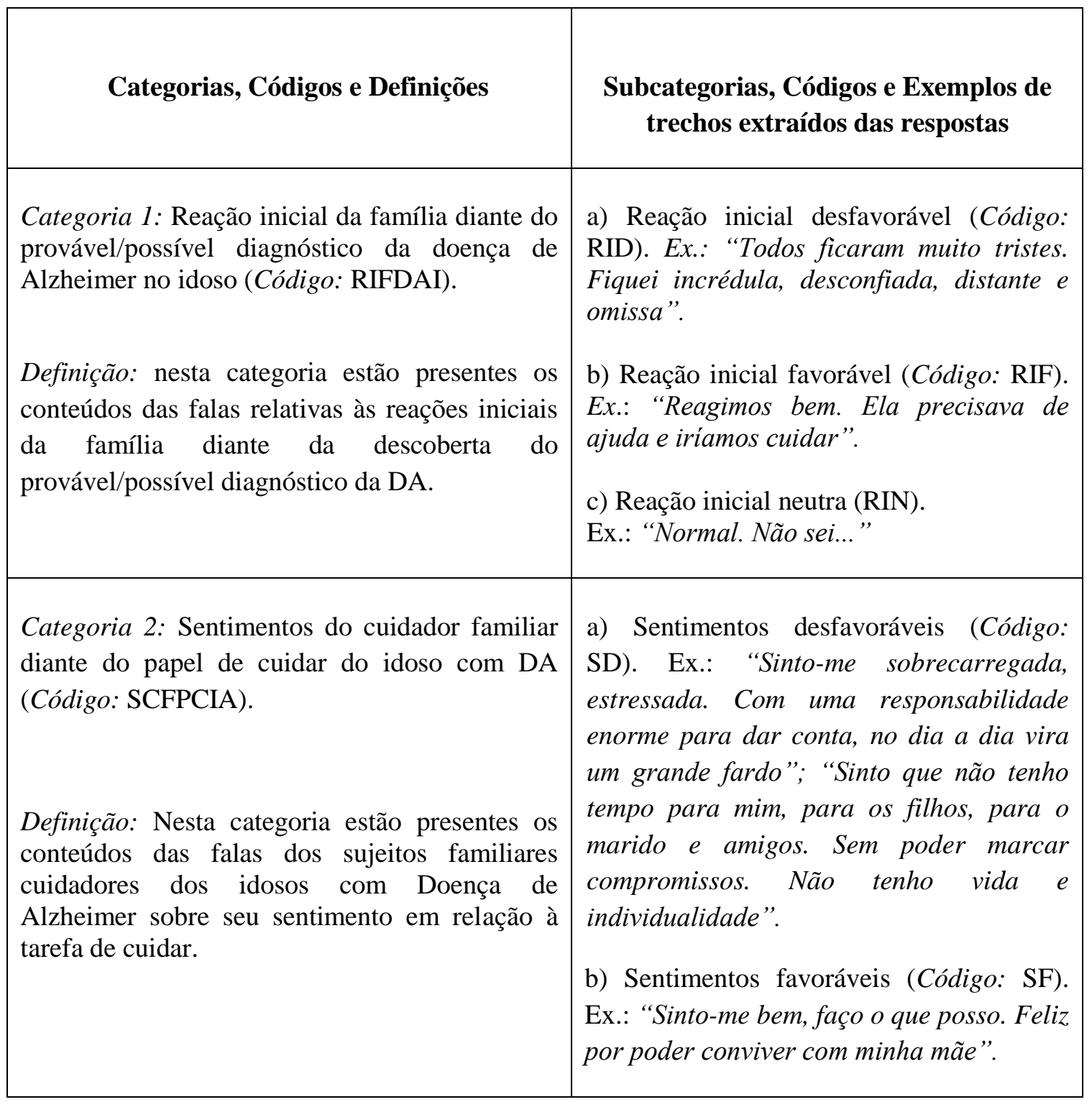




\section{Discussão}

O envelhecimento é um fenômeno diferencial e multifacetado para homens e mulheres, uma vez que, as mulheres apresentam maior longevidade quando comparadas aos homens (Gratão, 2010). Ao analisar a amostra de idosos com Alzheimer, percebeu-se uma predominância do sexo feminino (77\%) diante do sexo masculino (23\%). Já ambos os sexos apresentaram uma média de idade (sexo feminino, 83 anos e sexo masculino, 81 anos) superior à média de expectativa de vida revelada por alguns estudos internacionais, tais como os de Scazufca, Menezes, \& Almeida (2002) e Tooth, McKenna, Barnetti, Prescott, \& Murphy (2005).

Constatou-se ainda uma prevalência do sexo feminino (71\%) na função de cuidadoras, confirmando-se outros estudos realizados (Morais, 2004; Garcia, 2014). Tal fato pode ser explicado devido à construção sociocultural herdada pelas mulheres que, no passado, por não desempenharem funções fora do lar, eram as maiores responsáveis pelo cuidado da família e da casa. Nesse sentido, observa-se uma hierarquia da rede de apoio e tarefa de cuidar: em primeiro lugar, vem a esposa e, em seguida, vêm as filhas, especialmente, a mais velha (Silva, Passos, \& Barreto, 2012).

A tarefa do cuidar, que é considerada como responsabilidade doméstica, e desenvolvida no espaço privado, vem sofrendo, ao longo da história, constantes transformações, e adquirindo contornos diversos, conforme as particularidades econômicas e culturais predominantes em cada sociedade, território e época. Caldas (2000) indicou que a família apresenta necessidades que vão desde os aspectos materiais até os emocionais para o cuidado do idoso com Alzheimer. Um dos pontos mais importantes destacado pela autora é o suporte emocional e, para isso, é necessária uma rede de cuidados que ligue a família aos serviços de apoio e meios alternativos (tais como, instituições dedicadas ao idoso, e a ajuda formal ou informal no cuidado) que garantam qualidade de vida aos cuidadores principais.

A procura por esses serviços e meios alternativos, para o cuidado do idoso com Alzheimer, ficou evidenciada nos resultados da presente pesquisa, a qual constatou que a maioria dos familiares possuía, pelo menos, uma fonte de auxílio no cuidado, sendo a principal delas a AFAI, seguida pela ajuda informal de outros familiares. Também, a maioria apresentou um grau de satisfação positivo em relação ao auxílio recebido. Estudos indicaram que o auxílio nesse cuidado oferece aos idosos e suas famílias a possibilidade de prevenir institucionalizações precoces, hospitalizações e conflitos familiares (Durcharme, et al., 2011; Falcão, Teodoro, \& Bucher-Maluschke, 2016). 
Outrossim, na medida em que ocorre um investimento na formação e no fornecimento de informações aos cuidadores, espera-se uma melhoria na condução do caso, principalmente, na qualidade de vida das pessoas acometidas pela doença (Leite, Menezes, Lyra, \& Araújo, 2014).

A maioria dos familiares afirmou não ter tempo para cuidar de si mesmo, devido à quantidade de horas gastas no cuidado dedicado ao idoso com Alzheimer. Conforme Marins, Hansel, e Silva (2016), os familiares que residem com os idosos com DA diminuem seu tempo para visitar amigos e/ou relaxar, e têm maiores chances de apresentar episódios de depressão e se sentirem sobrecarregados. Também, na pesquisa de Falcão (2006), foram detectadas limitações e sobrecargas diante do papel de cuidar, observando-se o fenômeno conhecido como conflito de papel, ou seja, a ocorrência simultânea de dois ou mais conjuntos de pressões, de tal forma que o cumprimento de uma delas tornaria difícil ou mesmo impossível o cumprimento da outra.

Com a evolução da doença, o idoso depende cada vez mais de seu familiar para ajudá-lo a realizar suas Atividades Instrumentais da Vida Diária (AIVDs), ou seja, administrar sua vida dentro e fora do lar. As ações para realizar as AVDs e AIVDs são prejudicadas pela falta de memória ou pelo déficit no próprio conceito de ação de si, dificultando a aproximação das pessoas em suas relações afetivas, sociais e familiares. Sem a lembrança de fatos, lugares e pessoas, o idoso apresenta dificuldades de interação com o ambiente, perde a autonomia para cuidar de si, planejar e executar tarefas que permitem a adaptação psicossocial e a responsabilidade pelas próprias ações (Falcão, 2012). Assim sendo, as atividades instrumentais de cuidado apareceram com maior frequência em relação às atividades afetivas, sendo as principais delas: cuidar da alimentação/medicação, levar ao médico e controlar/orientar. Tal dado, também, foi detectado em outras pesquisas (Falcão, \& Bucher-Maluschke, 2008; Braz, 2014).

No presente estudo, as reações da família diante do provável/possível diagnóstico da DA foi em sua maioria desfavorável. Também, a maioria relatou a ocorrência de mudanças na dinâmica familiar após o surgimento da enfermidade, atingindo desfavoravelmente o relacionamento entre os membros. Esses resultados confirmaram dados sinalizados pela literatura, ou seja, que, durante o processo de cuidar, são desencadeadas tensões que podem gerar conflitos nas relações interpessoais. 
Conviver com o doente traz ao cuidador inúmeras sensações que podem vir a resultar em situações conflitantes. Grande parte dos familiares apresentaram dificuldades neste convívio, já que é preciso, antes de tudo, aceitar a doença para, em seguida, reconstruir o significado e a função do membro com DA na dinâmica familiar (Coelho, \& Alvim, 2004; Neumann, 2014). Destarte, para entender a complexidade de ser familiar e ao mesmo tempo cuidador de um idoso com a DA, faz-se necessário ampliar o foco para além da doença, buscando-se perceber o significado do processo de adoecimento de maneira multidimensional (Ilha, Zamberlan, Nicola, Araújo, \& Backes, 2014).

Sentimentos tais como, preocupação, tristeza e impaciência representam $10 \%$ do conteúdo de fala dos familiares. Diniz et al. (2015) salientaram que a doença de Alzheimer, além de causar situações de estresse ao cuidador, desgaste físico e emocional, também pode tornar a família disfuncional, levando o cuidador ao isolamento social, maior consumo de medicamentos e frequência às consultas médicas. Também, os gastos financeiros com essas consultas, medicações, e com a contratação de um cuidador em tempo integral, podem gerar sobrecarga e conflitos na família.

O sentimento de sobrecarga, vivenciado pelos cuidadores familiares investigados, deve-se, segundo a literatura, por diversos motivos, tais como: a) desgastes de ordem emocional, física e psicológica; b) pelo fato de o tratamento ser longo e de o paciente perder gradualmente suas funções cognitivas e evoluir para quadros de total dependência, exigindo, cada vez mais, a dedicação daqueles que com ele convivem; c) a idade mais avançada dos cuidadores, ou seja, daqueles com idade igual ou superior a 60 anos é um fator decisivo para maiores desgastes; d) residir com o idoso e cuidar dele a maior parte do tempo; e) dificuldades do cuidador em dividir igualitariamente as tarefas de cuidado com outros membros da família ou a ausência de revezamento com outros familiares, ocasionando pouco tempo para o autocuidado, descanso, lazer, vida afetiva, social e familiar; f) na maioria das vezes, o cuidado é realizado por apenas um membro da família, enquanto os demais "fingem" que tudo está bem e que a doença não existe, tornando a tarefa de cuidar ainda mais desgastante (Ilha, Zamberlan, Nicola, Araújo, \& Backes, 2014; Inouye, 2008; Inouye, \& Pedrazzani, 2010; Inouye, Pedrazzani, \& Pavarini, 2010; Lenardt, Willig, Seima, \& Pereira, 2011; Seima, Leinardt, \& Caldas, 2014).

A escassez de orientação a respeito da enfermidade pode interferir na forma como o cuidador planeja e executa as ações de cuidado. 
Assim sendo, a maneira de cuidar pode variar conforme a cultura de cada país, mas também de acordo com a educação em saúde. Essa educação pode favorecer o aprendizado, a empatia, a compreensão dos sintomas, ampliando oportunidades para os cuidadores resgatarem o próprio bem-estar físico e emocional, fortalecendo a autonomia e a tomada de decisão diante das demandas da enfermidade (Araújo, Oliveira, \& Pereira, 2012; Leite, Menezes, Lyra, \& Araújo, 2014);

\section{Considerações Finais}

Este estudo indicou que a doença de Alzheimer não atinge apenas o paciente, mas, também, toda a família, na sua complexidade, nas angústias geradas, nas dúvidas não esclarecidas. As atividades desenvolvidas findam, sobrecarregando os cuidadores que necessitam de um suporte para melhor lidar com essa situação. Faz-se mister que a doença de Alzheimer seja mais divulgada para que a sociedade e os profissionais de saúde adquiram conhecimentos sobre a doença, contribuindo para um diagnóstico precoce e uma melhor assistência. À medida que os profissionais se apropriam desse conhecimento podem atuar no cuidado direto e na educação e orientação dos cuidadores, familiares e idosos com DA.

Malgrado a limitação da amostra desta pesquisa, constataram-se aspectos vividos no cotidiano de familiares cuidadores, revelando dados que, em sua maioria, foram congruentes com a literatura. A partir disso, o presente estudo poderá fornecer fundamentações teóricas e práticas que visem a beneficiar os cuidadores formais e informais de idosos com Doença de Alzheimer. Para futuros estudos, recomenda-se investigar as relações conjugais de idosos em que um dos membros possui a DA e as relações entre avós com Alzheimer-filhos e netos.

\section{Referências}

Almeida, T. L. (2005). Características dos cuidadores de idosos dependentes no contexto da Saúde da Família. Dissertação de mestrado. Departamento de Medicina Social da Faculdade de Medicina de Ribeirão Preto da Universidade de São Paulo, Ribeirão Preto, SP, Brasil. 
Araújo, C. L. O., Oliveira, J. F., \& Pereira, J. M. (2012). Perfil de cuidadores de idosos com Doença de Alzheimer. São Paulo, SP: PUC-SP: Revista Kairós Gerontologia, 15(2), 109128.

Bardi, L. (1977/2000). Análise de conteúdo. Lisboa, Portugal: Edições 70.

Braz, M. C. (2014). Idosos com Doença de Alzheimer: Um Estudo sobre Cuidadores Formais e Informais. Trabalho de Conclusão de Curso, Graduação em Gerontologia, Universidade de São Paulo.

Caldas, C. P. (2000). Envelhecimento com dependência: responsabilidades e demandas da família. Cadernos de Saúde Pública, 19(3), 733-781. Recuperado em 01 julho, 2016, de: http://www.scielo.br/pdf/csp/v19n3/15880.pdf.

Coelho, G., \& Alvin, N. (2004). A dinâmica familiar, as fases do idoso com Alzheimer e os estágios vivenciados pela família na relação do cuidado no espaço domiciliar. Brasília, DF: Revista Brasileira de Enfermagem, 57(5), 541-544. Recuperado em 01 julho, 2016, de: http://dx.doi.org/10.1590/S0034-71672004000500005.

Diniz, S. O. S., Garcia, F. S., Sousa, L. J., Souza, D. C., Agathão, B. T. \& Eugenio, S. C. F. (2015). As dificuldades e os aspectos emocionais que envolvem os familiares/cuidadores. Revista Rede de Cuidados em Saúde, 01-12. Recuperado em 01 julho, 2016, de: http://publicacoes.unigranrio.br/index.php/rcs/article/view/1931.

Ducharme, F. C., Lévesque, L. L., Lachance, L. M., Kergoat, M. J., Legault, A. J., Beaudet, L. M., \& Zarit, S. H. (2011). "Learning to become a family caregiver" efficacy of an intervention program for caregivers following diagnosis of dementia in a relative. Gerontologist, 51(4), 484-494. Recuperado em 01 julho, 2016, de: doi: 10.1093/geront/gnr014.

Falcão, D. V. S. (2006). Doença de Alzheimer: um estudo sobre as filhas cuidadoras e suas relações familiares. Tese de doutorado, Universidade de Brasília. Brasília, DF.

Falcão, D. V. S., \& Bucher-Maluschke, J. S. N. F. (2008). Filhas que cuidam de pais/mães com provável/possível Doença de Alzheimer. Estudos de Psicologia, 13(3), 245-256. Recuperado em 01 julho, 2016, de: http://www.producao.usp.br/bitstream/handle/BDPI/2676/art_FALCAO_Filhas_que_cuidam _de_pais-maes_com_provavel-possivel_2008.pdf?sequence=1\&isAllowed=y.

Falcão, D. V. S.. \& Bucher-Maluschke, J. S. N. F. (2009). Cuidar de Familiares Idosos com a doença de Alzheimer: uma reflexão sobre aspectos psicossociais. Psicologia em Estudo, 14(4), 777-786. Recuperado em 01 julho, 2016, de: http://www.scielo.br/pdf/pe/v14n4/v14n4a18.

Falcão, D. V. S. (2012). Atenção psicogerontológica, sociofamiliar e educativa dos cuidadores e familiares de idosos com doença de Alzheimer. In: Nunes, P.V., Falcão, D. V. S., Cachioni, M., \& Forlenza, O. V. Doença de Alzheimer - Uma Perspectiva do Tratamento Multiprofissional, 137-154. São Paulo, SP: Atheneu.

Falcão, D. V. S., Teodoro, M. L. M., \& Bucher-Maluschke, J. S. N. F. (2016). Family cohesion: A study on caregiving daughters of parents with Alzheimer's disease. Interpersona: an International Journal on Personal Relationships, 10(Supp.1), 61-74. Recuperado em 01 julho, 2016, de: doi:10.5964/ijpr.v10isupp1.244. 
Figueiredo, D., \& Sousa, L.(2008). Percepção do estado de saúde e sobrecarga em cuidadores familiares de idosos dependentes com e sem demência. Revista Portuguesa de Saúde Pública, 26(1). Recuperado em 01 julho, 2016, de: https://www.ensp.unl.pt/dispositivos-de-apoio/cdi/cdi/sector-de-publicacoes/revista/ 20002008/pdfs/rpsp-1-2008/02_1-2008.pdf.

Freitas, I., Paula, K., Soares, J., \& Parente, A. (2008). Convivendo com o portador de Alzheimer: perspectivas do familiar cuidador. Revista Brasileira de Enfermagem, 61(4), 508-513. Recuperado em 01 julho, 2016, de: http://www.scielo.br/pdf/reben/v61n4/18.pdf.

Garcia, C. R. (2014). Cuidadores Familiares de Idosos com Doença de Alzheimer: Um Estudo sobre Estresse Percebido e Funcionamento Familiar. Trabalho de Conclusão de Curso (Graduação em Gerontologia), Universidade de São Paulo.

Gratão, A. C. M. (2006). Demanda do Cuidador Familiar com Idoso Demenciado. Dissertação de mestrado em Enfermagem Fundamental. Escola de Enfermagem de Ribeirão Preto, Universidade de São Paulo, Ribeirão Preto, SP, Brasil.

Gratão, A. C. M. (2010). Sobrecarga Vivenciada por Cuidadores de Idosos na Comunidade. Tese de doutorado em Enfermagem Fundamental. Escola de Enfermagem de Ribeirão Preto, Universidade de São Paulo, Ribeirão Preto, SP, Brasil.

Ilha, S., Zamberlan, C., Nicola, G. D. O., Araújo, A. S., \& Backes, D. S. (2014). Refletindo acerca da doença de Alzheimer no contexto familiar do idoso: implicações para a Enfermagem. Revista de Enfermagem do Centro Oeste Mineiro, 4(1), 1057-1065. Recuperado em 01 julho, 2016, de: doi: http://dx.doi.org/10.19175/recom.v0i0.378.

Inouye, K. (2008). Educação, qualidade de vida e doença de Alzheimer: visões de idosos e seus familiares. Dissertação de mestrado em Educação Especial, Universidade Federal de São Carlos, São Carlos, SP, Brasil.

Inouye, K., \& Pedrazzani, E. S. (2010). Influência da doença de Alzheimer na percepção de qualidade de vida do idoso. Caderno de Saúde Pública, 4(44), 1093-1099.

Inouye, K., Pedrazzani, E. S., \& Pavarini, S. C. I. (2010). Implicações da doença de Alzheimer na qualidade de vida do cuidador: um estudo comparativo. Caderno de Saúde Pública, 26(5), 891-899. Recuperado em 01 julho, 2016, de: http://www.scielo.br/pdf/csp/v26n5/11.pdf.

Kang, H. S., Myung, W., Na, D. L., Kim, S. Y., Lee, J. H., Han, S. H., Choi, S. H., Kim, S. Y., Kim, S., \& Kim, D. K. (2014). Factors Associated with caregiver burden in patients with Alzheimer's Disease. Psychiatry Investigation, 11(2), 152-159. Recuperado em 01 julho, 2016, de: doi: 10.4306/pi.2014.11.2.152.

Leite, C. D. S. M., Menezes, T. L. M., Lyra, E. V. V., \& Araújo, C. M. T. (2014). Conhecimento e intervenção do cuidador na doença de Alzheimer: uma revisão da literatura. Jornal Brasileiro de Psiquiatria, 63(1), 48-56. Recuperado em 01 julho, 2016, de: http://dx.doi.org/10.1590/0047-2085000000007.

Lenardt, M. H., Willig, M. H., Seima, M. D., \& Pereira, L. F. (2011). A condição de saúde e satisfação com a vida do cuidador familiar de idoso com Alzheimer. Colombia Médica, 42(2), 17-25. Recuperado em 01 julho, 2016, de: http://www.redalyc.org/pdf/283/28322504003.pdf.

Machado, J. C. B. (2012). Doença de Alzheimer. In: Tratado de Geriatria e Gerontologia. ( $2^{\mathrm{a}}$ ed., cap. 25). Rio de Janeiro, RJ: Guanabara Koogan. 
Marins, A. M. F., Hansel, C. G., \& Silva, J. (2016). Mudanças de comportamento em idosos com Doença de Alzheimer e sobrecarga para o cuidador. Escola Anna Nery, 20(2), 352-356. Recuperado em 01 julho, 2016, de: http://www.scielo.br/pdf/ean/v20n2/1414-8145-ean-2002-0352.pdf.

Maslach, C., Schaufeli, W. B., \& Leiter, M. P. (2001). Job Bounout. Annual Review Psychology, 52, 397-422. Recuperado em 01 julho, 2016, de: http://www.wilmarschaufeli.nl/publications/Schaufeli/154.pdf.

Moon, H., \& Dilworth-Anderson, P. (2014). Baby boomer caregiver and dementia caregiving: findings from the National Study of Caregiving. Age and Ageing, 44(2), 300306. Recuperado em 01 julho, 2016, de: doi: 10.1093/ageing/afu119.

Morais, H. (2004). Representação sobre o cuidado e a qualidade de vida do cuidador de idosos dependentes no Programa de Saúde da Família de Uberaba, MG. Dissertação de mestrado, Departamento de Medicina Social da Faculdade de Medicina de Ribeirão Preto, Universidade de São Paulo, Ribeirão Preto, SP, Brasil.

Neri, A. L., \& Sommerhalder, C. (2002). As várias faces do cuidado e do bem-estar do cuidado. In: Neri, A. L. (Org.). Cuidar de idosos no contexto da família: questões psicológicas e sociais. Campinas, SP: Atheneu.

Neumann, S. M. F. (2014). Doença de Alzheimer: Intervenção psicoeducativa para familiares cuidadores. Tese de doutorado em Psicologia Clínica. Recife, PE: Universidade Católica de Pernambuco.

Neumann, S. M. F., Dias, C. M. S. B., \& Falcão, D. V. S. (2016). Familiares cuidadores de idosos com doença de Alzheimer: a importância de cuidar de quem cuida. In: Falcão, D. V. S., Araújo, L. F., \& Pedrosa, J. S. (Orgs.). Velhices: temas emergentes nos contextos psicossocial e familiar, 189-206. Campinas, SP: Alínea.

Oliveira, J. S. C., Ferreira, A. O. M., Fonseca, A. M., \& Paes, G. O. (2016). Desafios de cuidadores familiares de idosos com doença de Alzheimer inseridos em um grupo de apoio. Revista de Enfermagem UFPE, 10(2), 539-544. Recuperado em 01 julho, 2016, de: http://saudepublica.bvs.br/pesquisa/resource/pt/bde-28275.

Resende, M. C. F., \& Dias, E. C. (2008). Cuidadores de idosos: um novo / velho trabalho. Physis: Revista de Saúde Coletiva, 18(4), 785-800. Recuperado em 01 julho, 2016, de: $\mathrm{http} / / /$ bases.bireme.br/cgi-bin/wxislind.exe/iah/online/?IsisScript=iah/iah.xis\&src=google \& base $=$ LILACS\&lang=p\&nextAction=lnk\&exprSearch= 519692 \&indexSearch=ID.

Scazufca, M., Menezes, P. R., \& Almeida, O. P. (2002). Caregivers burden in elderly population with depression in São Paulo, Brazil. Social Psychiatry and Psychiatric Epidemiology, 37(Issue 9), 416-422. Recuperado em 01 julho, 2016, de: doi: 10.1007/s00127-002-0571-6.

Schulz, R., \& Beach, S. (1999). Caregiving as a risk factor for mortality: the caregiver health. Journal of American Medical Association, 282, 2215-2224. Recuperado em 01 julho, 2016, de: https://www.ncbi.nlm.nih.gov/pubmed/10605972.

Seima, M. D., Lenardt, M. H., \& Caldas, C. P. (2014). Relação no cuidado entre o cuidador familiar e o idoso com Alzheimer. Revista Brasileira de Enfermagem, 67(2), 233-240. Recuperado em 01 julho, 2016, de: http://www.scielo.br/pdf/reben/v67n2/0034-7167-reben67-02-0233.pdf. 
Silva, C. F., Passos, V. M. A., \& Barreto, S. M. (2012). Frequência e repercussão da sobrecarga de cuidadoras familiares de idosos com demência. Revista Brasileira de Geriatria e Gerontologia, 15(4), 707-731. Recuperado em 01 julho, 2016, de: http://www.scielo.br/pdf/rbgg/v15n4/11.pdf.

Tooth, L., McKenna, K., Barnetti, A., Prescott, C., \& Murphy, S. (2005). Caregivers burden, time spent caring and health status in the first 12 months following stroke. Brain Injury, 19(12), 963-974. Recuperado em 01 julho, 2016, de: https://www.ncbi.nlm.nih.gov/pubmed/16263639.

Vilela, L., \& Caramelli, P. (2006). A doença de Alzheimer na visão de familiares de pacientes. Revista da Associação Médica Brasileira, 52(3), 148-152. Recuperado em 01 julho, 2016, de: http://bases.bireme.br/cgi-bin/wxislind.exe/iah/online/?IsisScript =iah /iah.xis\&src=google \&base=LILACS\&lang=p\&nextAction=lnk\&exprSearch=431170\&inde $\mathrm{xSearch}=\mathrm{ID}$.

Recebido em 29/01/2017

Aceito em 30/03/2017

Camila Rodrigues Garcia - Bacharel em Gerontologia, Universidade de São Paulo, USP. Escola de Artes, Ciências e Humanidades, EACH. Mestranda em Gerontologia pela USP, $\mathrm{EACH}$

E-mail: camila.rodrigues.usp@gmail.com

Gabriela Cabett Cipolli - Bacharel em Gerontologia, Universidade de São Paulo, USP. Escola de Artes, Ciências e Humanidades, EACH. Mestranda em Gerontologia pela USP, $\mathrm{EACH}$.

E-mail: gabicipolli@ hotmail.com

Jéssica Pucci dos Santos - Bacharel em Gerontologia, Universidade de São Paulo, USP. Escola de Artes, Ciências e Humanidades, EACH.

E-mail: jessica.pucci.santos@gmail.com

Luca Pasquali Freitas - Bacharel em Gerontologia, Universidade de São Paulo, USP. Escola de Artes, Ciências e Humanidades, EACH.

E-mail: luca.pasquali11@gmail.com 
Mayara Cartoni Braz - Bacharel em Gerontologia, Universidade de São Paulo, USP. Escola de Artes, Ciências e Humanidades, EACH.

E-mail: mayaracartoni.braz@gmail.com

Deusivania Vieira da Silva Falcão - Psicóloga e Mestra em Psicologia Social, UFPB. Doutora em Psicologia, UnB. Professora Doutora nos Cursos de Graduação e PósGraduação em Gerontologia, Universidade de São Paulo, USP. Escola de Artes, Ciências e Humanidades, EACH.

E-mail: deusivaniafalcao@gmail.com 